\title{
Edaphic, structural and physiological contrasts across Amazon Basin forest- savanna ecotones suggest a role for potassium as a key modulator of tropical woody vegetation structure and function
}

\section{J. Lloyd et al.}

Correspondence to: J. Lloyd (jonathan.lloyd@imperial.ac.uk)

The copyright of individual parts of the supplement might differ from the CC-BY 3.0 licence. 


\begin{tabular}{|c|c|c|c|c|c|c|c|c|c|c|c|c|c|c|c|c|c|c|c|c|}
\hline \multirow{2}{*}{$\begin{array}{c}\text { PLOT } \\
\text { TUC-01 }\end{array}$} & \multirow{2}{*}{$\mathbb{W}$} & \multirow{2}{*}{$\begin{array}{c}\boldsymbol{T}_{\mathrm{A}} \\
\left({ }^{\circ} \mathbf{C}\right) \\
24.8\end{array}$} & \multirow{2}{*}{$\begin{array}{c}\begin{array}{c}\boldsymbol{P}_{\mathrm{A}} \\
\left(\mathbf{m ~ a}^{-1}\right)\end{array} \\
0.82\end{array}$} & \multirow{2}{*}{$\begin{array}{c}\begin{array}{c}\boldsymbol{\theta} \\
\left(\mathbf{m}^{3} \mathbf{m}^{-3}\right)\end{array} \\
0.21\end{array}$} & \multicolumn{2}{|c|}{$\begin{array}{l}d_{\mathbf{R}} \\
\\
(\mathbf{m})\end{array}$} & \multirow{2}{*}{$\begin{array}{c}\mathbf{p H} \\
6.1\end{array}$} & \multirow{2}{*}{$\begin{array}{c}\text { clay } \\
0.18\end{array}$} & \multirow{2}{*}{$\begin{array}{r}\text { sand } \\
0.48\end{array}$} & \multirow{2}{*}{$\begin{array}{c}\text { Silt } \\
0.35\end{array}$} & \multicolumn{2}{|c|}{$\begin{array}{c}{[\mathrm{N}]_{\mathrm{s}} \quad[\mathrm{C}]_{\mathrm{s}}} \\
\mathrm{g} \mathrm{kg}^{-1}\end{array}$} & \multirow{2}{*}{$\begin{array}{c}{[\mathbf{A l}]_{\text {ex }}} \\
0.3\end{array}$} & \multicolumn{3}{|c|}{$\begin{array}{rrr}{[\mathrm{Ca}]_{\mathrm{ex}}} & {[\mathrm{K}]_{\mathrm{ex}}} & {[\mathrm{Mg}]_{\mathrm{ex}}} \\
& \mathrm{mmol} \mathrm{eq}^{+} \mathbf{k g}^{-1}\end{array}$} & \multirow{2}{*}{$\frac{[\mathrm{Na}]_{\mathrm{ex}}}{2.3}$} & \multicolumn{2}{|c|}{$\begin{array}{c}P_{\text {extr }} \quad P_{\text {resid }} \\
\text { mg kg }^{-1} \\
\end{array}$} & \multirow{2}{*}{$\begin{array}{r}\text { WRB Classification } \\
\text { Haplic Cambisol (Hypereutric, Greyic, Siltic) }\end{array}$} \\
\hline & & & & & 1.4 & 0.29 & & & & & 1.22 & 10.76 & & 28.2 & 1.9 & 18.8 & & 299.6 & 237.5 & \\
\hline TUC-03 & $\mathbb{S}$ & 24.7 & 0.89 & 0.18 & 1.0 & 0.18 & 6.4 & 0.04 & 0.78 & 0.18 & 0.54 & 6.16 & 0.6 & 16.4 & 0.6 & 4.6 & 0.1 & 58.4 & 50.6 & \\
\hline OTT-01 & $\mathbb{F}$ & 24.6 & 1.15 & 0.17 & 1.9 & 0.32 & 5.9 & 0.10 & 0.75 & 0.14 & 1.15 & 10.75 & 0.4 & 12.2 & 0.7 & 4.8 & 0.1 & 80.0 & 143.4 & Plinthic Acrisol (Epieutric, Epiarenic) \\
\hline OTT-02 & $\mathbb{S}$ & 24.6 & 1.15 & 0.18 & 4.0 & 0.74 & 5.1 & 0.19 & 0.71 & 0.10 & 0.49 & 5.46 & 2.0 & 3.3 & 0.3 & 1.8 & 0.1 & 49.5 & 50.3 & Haplic Ferralsol (Dystric, Xanthic) \\
\hline OTT-03 & $\mathbb{S}$ & 24.6 & 1.15 & 0.18 & 2.0 & 0.39 & 5.2 & 0.17 & 0.65 & 0.18 & 1.15 & 15.78 & 1.0 & 10.3 & 0.6 & 5.2 & 0.1 & 67.9 & 62.2 & Umbric Ferralsol (Dystric) \\
\hline ACU-01 & IF & 24.1 & 1.27 & 0.12 & 2.0 & 0.24 & 6.3 & 0.31 & 0.49 & 0.20 & 1.74 & 18.48 & 0.9 & 15.0 & 1.2 & 5.1 & 0.0 & 198.6 & 308.3 & Nitic Acrisol (Epieutric, Chromic) \\
\hline LFB-03 & $\mathbb{S}$ & 24.1 & 1.44 & 0.13 & 1.9 & 0.25 & 4.9 & 0.32 & 0.50 & 0.19 & 0.96 & 13.69 & 3.7 & 0.1 & 0.3 & 0.3 & 0.2 & 110.7 & 73.3 & Geric Acric Gibbsic Ferralsol (Dystric) \\
\hline LFB-01 & IF & 23.9 & 1.46 & 0.17 & 4.0 & 0.68 & 4.6 & 0.20 & 0.74 & 0.06 & 0.83 & 10.85 & 10.9 & 0.2 & 0.7 & 0.6 & 0.0 & 46.6 & 60.5 & Geric Acric Ferralsol (Dystric) \\
\hline NXV-01 & $\mathbb{S}$ & 25.0 & 1.50 & 0.12 & 1.9 & 0.23 & 4.6 & 0.11 & 0.85 & 0.03 & 0.38 & 6.07 & 8.4 & 1.0 & 1.0 & 1.5 & 0.1 & 65.5 & 39.0 & Vetic Acric Ferralsol (Alumic, Hyperdystric, Arenic, Xanthic) \\
\hline NXV-02 & $\mathbb{S}$ & 25.0 & 1.50 & 0.15 & 4.0 & 0.60 & 4.5 & 0.18 & 0.75 & 0.07 & 0.84 & 12.83 & 13.6 & 1.7 & 1.2 & 2.3 & 0.0 & 88.2 & 39.2 & Vetic Acric Ferralsol (Alumic, Hyperdystric, Epiarenic) \\
\hline VCR-01 & IF & 25.3 & 1.51 & 0.11 & 4.0 & 0.45 & 4.2 & 0.45 & 0.47 & 0.08 & 1.05 & 15.92 & 17.3 & 1.5 & 1.7 & 1.4 & 0.2 & 123.3 & 192.7 & Geric Ferralsol (Alumic, Hyperdystric, Clayic, Rhodic) \\
\hline VCR-02 & $\mathbb{F}$ & 25.2 & 1.51 & 0.14 & 4.0 & 0.54 & 4.7 & 0.24 & 0.66 & 0.10 & NA & 10.61 & 5.3 & 3.6 & 1.1 & 2.6 & 0.1 & 81.1 & 77.2 & Geric Plinthic Ferralsol (Alumic, Hyperdystric, Endoclayic, Rhodic) \\
\hline SMT-03 & $\mathbb{S}$ & 25.8 & 1.60 & 0.14 & 4.0 & 0.48 & 4.8 & 0.04 & 0.93 & 0.03 & 0.23 & 3.53 & 4.9 & 0.1 & 0.2 & 0.1 & 0.1 & 25.3 & 10.7 & Hypoluvic Ferralic Arenosol (Hyperdystric) \\
\hline SMT-01 & $\mathbb{S}$ & 25.8 & 1.60 & 0.14 & 4.0 & 0.55 & 4.9 & 0.06 & 0.91 & 0.03 & 0.25 & 4.38 & 4.7 & 0.1 & 0.3 & 0.2 & 0.1 & 20.3 & 10.7 & Hypoluvic Ferralic Arenosol (Hyperdystric) \\
\hline SMT-02 & $\mathbb{S}$ & 25.8 & 1.60 & 0.12 & 4.0 & 0.55 & 4.6 & 0.05 & 0.91 & 0.04 & 0.24 & 3.92 & 5.0 & 0.2 & 0.4 & 0.2 & 0.1 & 22.4 & 21.0 & Hypoluvic Ferralic Arenosol (Hyperdystric) \\
\hline FLO-01 & $\mathbb{F}$ & 25.5 & 1.61 & 0.14 & 4.0 & 0.57 & 4.2 & 0.16 & 0.78 & 0.06 & 0.62 & 9.03 & 4.3 & 0.3 & 0.5 & 0.4 & 0.2 & 51.9 & 65.1 & Geric Ferralsol (Alumic, Hyperdystric, Epiarenic, Rhodic) \\
\hline TAN-01 & IF & 25.1 & 1.65 & 0.11 & 4.0 & 0.45 & 4.1 & 0.49 & 0.46 & 0.05 & 1.57 & 25.48 & 17.2 & 0.5 & 0.7 & 0.9 & 0.3 & 89.2 & 52.3 & Hypoluvic Ferralic Arenosol (Hyperdystric) \\
\hline TAP-123 & IF & 25.9 & 1.88 & 0.11 & 4.0 & 0.45 & 3.8 & 0.66 & 0.23 & 0.10 & 1.20 & 16.40 & 17.4 & 1.7 & 0.6 & 1.5 & 0.3 & 35.6 & 43.3 & Geric Ferralsol (Alumic, Hyperdystric, Clayic, Xanthic) \\
\hline TAP-04 & $F$ & 25.1 & 1.96 & 0.11 & 4.0 & 0.45 & 3.8 & 0.89 & 0.02 & 0.08 & 1.67 & 23.59 & 23.3 & 3.0 & 2.2 & 0.7 & 0.4 & 67.6 & 124.6 & Typic Ferralsol (Alumic, Hyperdystric, Clayic) \\
\hline ALC-02 & $\mathbb{S}$ & 26.0 & 1.97 & 0.05 & 4.0 & 0.21 & 4.8 & 0.05 & 0.91 & 0.04 & 0.49 & 10.24 & 6.9 & 0.2 & 0.3 & 0.3 & 0.2 & 66.9 & 49.9 & Hyperalbic Arenosol (Alumic, Hyperdystric) \\
\hline ALC-01 & $\mathbb{S}$ & 25.9 & 2.02 & 0.05 & 3.5 & 0.18 & 5.1 & 0.02 & 0.97 & 0.01 & 0.21 & 4.20 & 1.6 & 0.2 & 0.1 & 0.2 & 0.0 & 24.9 & 16.7 & Hyperalbic Arenosol (Alumic, Hyperdystric) \\
\hline
\end{tabular}

Table S1. Climatic and edaphic characteristics of the study sites(ordered according to mean annual precipitation): Abbreviations used: $\mathbb{V}$ - Vegetation formation type; $T_{\mathrm{A}}$ - mean annual temperature; $P_{\mathrm{A}}$ mean annual precipitation; $\theta$-soil water storage capacity (averaged across rooting depth); $d_{\mathrm{R}}-$ depth of rooting; $\theta_{\mathrm{P}}-$ maximum plant available water in soil profile; $\mathrm{pH}$ - soil $\mathrm{pH},[\mathrm{C}]_{\mathrm{s}}$ - soil carbon concentration; $[\mathrm{N}]_{\mathrm{s}}-$ soil nitrogen concentration; [ ] $]_{\mathrm{ex}}-$ soil exchangeable cations, $[\mathrm{P}]_{\text {extr }}$ - soil extractable phosphorus pool, $[\mathrm{P}]_{\text {resid }}$ - soil residual phosphorus pool; WRB - World Reference Base. Soil chemical and textural values represent the top $0.3 \mathrm{~m}$ of soil. For ease of interpretation forest plots are in black and savanna sites in red. Plots of similar location and climate have been grouped together with either grey or absent shading to easier enable forest-savanna comparisons. Other site details (for example precise co-ordinates and elevation) are provided in the supplementary information of Veenendaal et al. (2014). 


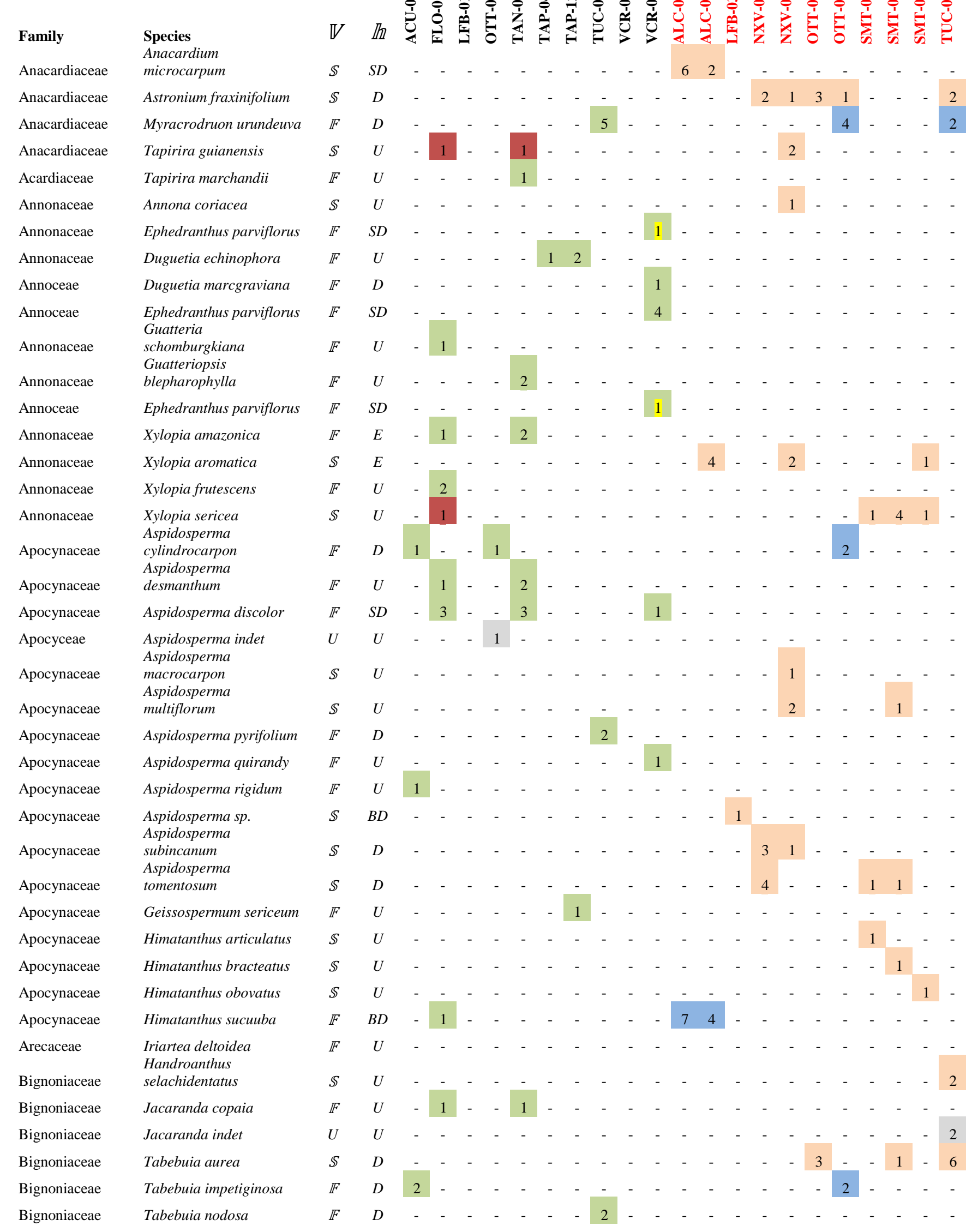




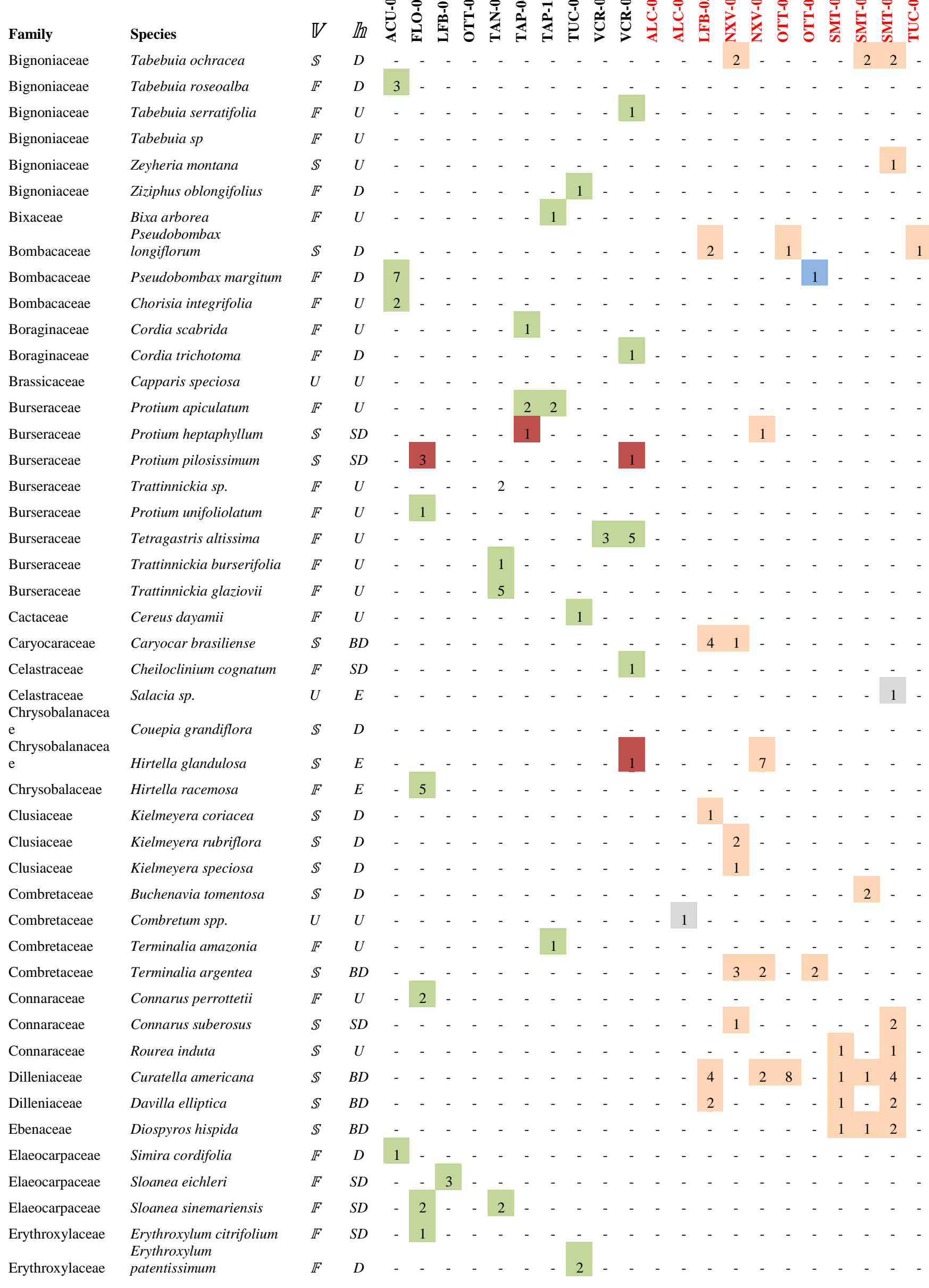




\begin{tabular}{|c|c|c|c|c|c|c|c|c|c|c|c|c|c|c|c|c|c|c|c|c|c|c|c|c|}
\hline Family & Species & 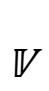 & Ill & 官 & 豆 & 量 & مَ" & 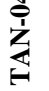 & $\sum_{\substack{1 \\
\vdots}}^{1}$ & $\sum^{\frac{1}{1}}$ & లِ & غُ & نَّ & Uె & Uి & 先 & $\begin{array}{l}0 \\
1 \\
z \\
z\end{array}$ & 章 & 'ְ & 01 & 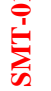 & 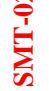 & & తి \\
\hline Erythroxylaceae & Erythroxylum sp. & $U$ & $U$ & - & - & - & - & - & - & - & - & - & - & - & 1 & - & - & - & - & - & - & - & & - \\
\hline Euphorbiaceae & $\begin{array}{l}\text { Alchornea schomburgkii } \\
\text { Chaetocarpus }\end{array}$ & $\mathcal{S}$ & $U$ & - & - & - & - & - & - & - & - & - & - & - & - & - & - & 1 & - & - & - & - & & - \\
\hline Euphorbiaceae & echinocarpus & $\mathbb{F}$ & $E$ & - & - & - & - & 3 & - & - & - & - & 6 & - & - & - & - & 1 & - & - & - & - & - & - \\
\hline Euphorbiaceae & Hyeronima oblonga & $\mathbb{F}$ & $U$ & - & - & 3 & - & - & - & - & - & - & - & - & - & - & - & - & - & - & - & - & & \\
\hline Euphorbiaceae & Mabea fistulifera & $\mathbb{F}$ & $S D$ & - & - & - & - & - & - & - & - & - & 1 & - & - & - & - & - & - & - & - & - & & \\
\hline Euphorbiaceae & Maprounea guianensis & $\mathbb{F}$ & $D$ & - & - & - & - & - & - & - & - & - & - & - & - & - & - & 1 & - & - & - & 1 & & \\
\hline Euphorbiaceae & Pera coccinea & $\mathbb{F}$ & $U$ & - & 3 & - & - & 1 & - & - & - & - & 1 & - & - & - & - & - & - & - & - & - & - & - \\
\hline Euphorbiaceae & Sapium longifolium & $\mathbb{F}$ & $U$ & - & - & - & - & - & - & - & - & - & 2 & - & - & - & - & - & - & - & - & - & - & - \\
\hline Euphorbiaceae & Sapium spp. & $U$ & $U$ & - & - & - & - & - & - & - & - & - & - & - & 3 & - & - & - & - & - & - & - & - & - \\
\hline Fabaceae & Abarema mataybifolia & $\mathbb{F}$ & $U$ & - & - & - & - & - & 1 & - & - & - & - & - & - & - & - & - & - & - & - & - & - & - \\
\hline Fabaceae & Acacia tenuifolia & $\mathbb{F}$ & $D$ & - & - & - & 2 & - & - & - & - & - & - & - & - & - & - & - & - & - & - & - & - & - \\
\hline Fabaceae & Acosmium cardesii & $\mathbb{F}$ & $E$ & - & - & - & 8 & & - & - & - & - & - & - & - & - & - & - & - & - & - & - & - & - \\
\hline Fabaceae & Diplotropis purpurea & $\mathbb{F}$ & $E$ & & & & & 2 & & & & & & & & & & & & & & & & \\
\hline Fabaceae & Adenanthera colubrina & $\mathbb{F}$ & $D$ & 2 & - & - & 3 & - & - & - & 5 & - & - & - & - & - & - & - & - & - & - & - & - & - \\
\hline Fabaceae & Amburana cearensis & $\mathbb{F}$ & $U$ & 2 & - & - & - & - & - & - & - & - & - & - & - & - & - & - & - & - & - & - & - & - \\
\hline Fabaceae & Andira vermifuga & $\mathcal{S}$ & $D$ & - & - & - & - & - & - & - & - & - & - & - & - & - & - & - & - & - & - & - & & 1 \\
\hline Fabaceae & Apuleia leiocarpa & $\mathbb{F}$ & $U$ & - & - & - & - & - & - & - & - & 1 & - & - & - & - & - & - & - & - & - & - & & - \\
\hline Fabaceae & Bauhinia rufa & $\mathcal{S}$ & $U$ & - & - & - & - & - & - & - & - & - & - & - & - & - & - & - & - & - & - & - & & 1 \\
\hline Fabaceae & Bowdichia virgilioides & $\mathcal{S}$ & $D$ & - & - & - & - & - & - & - & - & - & - & - & - & 1 & - & 1 & - & 1 & - & 1 & & 1 \\
\hline Fabaceae & $\begin{array}{l}\text { Caesalpinia pluviosa } \\
\text { Centrolobium }\end{array}$ & $\mathbb{F}$ & $B D$ & - & - & - & 2 & - & - & - & - & - & - & - & - & - & - & - & - & - & - & - & & - \\
\hline Fabaceae & microchaete & $\mathbb{F}$ & $D$ & - & - & - & 2 & - & - & - & - & - & - & - & - & - & - & - & - & - & - & - & - & - \\
\hline Fabaceae & Copaifera duckei & $\mathbb{F}$ & $U$ & - & - & - & - & - & 9 & - & - & - & - & - & - & - & - & - & - & - & - & - & - & - \\
\hline Fabaceae & Copaifera martii & $\mathcal{S}$ & $B D$ & - & - & - & - & - & - & - & - & - & - & 3 & 1 & - & - & - & - & - & 1 & - & & 1 \\
\hline Fabaceae & Dalbergia miscolobium & $\mathcal{S}$ & $B D$ & - & - & - & - & - & - & - & - & - & - & - & - & - & - & - & - & - & - & - & & - \\
\hline Fabaceae & Dimorphandra mollis & $\mathcal{S}$ & $B D$ & - & - & - & - & - & - & - & - & - & - & - & - & - & - & - & 3 & - & - & - & & - \\
\hline Fabaceae & Dipteryx alata & $\mathcal{S}$ & $U$ & - & - & - & - & - & - & - & - & - & - & - & - & - & - & 1 & - & 2 & - & - & & - \\
\hline Fabaceae & Diptychandra aurantiaca & $\mathcal{S}$ & $D$ & - & - & - & - & - & - & - & - & - & - & - & - & 1 & - & - & - & 3 & - & - & & - \\
\hline Fabaceae & $\begin{array}{l}\text { Dussia tessmannii } \\
\text { Enterolobium }\end{array}$ & $\mathbb{F}$ & $U$ & - & - & - & - & - & - & - & - & - & - & - & - & - & - & - & - & - & - & - & - & - \\
\hline Fabaceae & gummiferum & $\mathcal{S}$ & $D$ & - & - & - & - & - & - & - & - & - & - & - & - & - & - & - & - & - & - & - & & - \\
\hline Fabaceae & Hymenaea courbaril & $\mathbb{F}$ & $E$ & - & - & - & - & - & - & - & - & 2 & 1 & - & - & - & - & - & - & 1 & - & - & - & - \\
\hline Fabaceae & Hymenaea stigonocarpa & $\mathcal{S}$ & $B D$ & - & - & - & - & - & - & - & - & - & - & - & - & - & 1 & 1 & - & - & 1 & 1 & 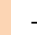 & - \\
\hline Fabaceae & Inga heterophylla & $\mathbb{F}$ & $E$ & - & 2 & - & - & - & - & - & - & - & - & - & - & - & - & - & - & - & - & - & & - \\
\hline Fabaceae & Inga microcalyx & $\mathbb{F}$ & $U$ & - & - & - & - & - & 1 & - & - & - & - & - & - & - & - & - & - & - & - & - & & - \\
\hline Fabaceae & Inga thibaudiana & $\mathbb{F}$ & $E$ & - & - & - & - & - & - & - & - & - & 1 & - & - & - & - & - & - & - & - & - & & - \\
\hline Fabaceae & Luetzelburgia praecox & $\mathcal{S}$ & $U$ & - & - & - & - & - & - & - & - & - & - & - & - & - & - & 2 & - & - & - & - & & - \\
\hline Fabaceae & Machaerium acutifolium & $\mathbb{F}$ & $S D$ & - & - & - & 1 & - & - & - & - & - & - & - & - & - & - & - & - & - & - & - & & - \\
\hline Fabaceae & Machaerium opacum & $\mathcal{S}$ & $S D$ & - & - & - & - & - & - & - & - & - & - & - & - & - & - & - & - & - & - & - & & - \\
\hline Fabaceae & Machaerium scleroxylon & $\mathbb{F}$ & $S D$ & - & - & - & 1 & - & - & - & - & - & - & - & - & - & - & - & - & - & - & - & & - \\
\hline Fabaceae & Machaerium villosum & $\mathbb{F}$ & $U$ & 1 & - & - & - & - & - & - & - & - & - & - & - & - & - & - & - & - & - & - & & - \\
\hline Fabaceae & Ormosia paraensis & $\mathbb{F}$ & $U$ & - & 2 & - & - & - & - & - & - & - & - & - & - & - & - & - & - & - & - & - & & - \\
\hline Fabaceae & Peltogyne confertiflora & $\mathcal{S}$ & $B D$ & - & - & - & - & - & - & - & - & - & - & - & - & - & - & - & - & - & - & 2 & & - \\
\hline Fabaceae & Piptadenia viridiflora & $\mathbb{F}$ & $U$ & - & - & - & 2 & - & - & - & - & - & - & - & - & - & - & - & - & - & - & - & & - \\
\hline Fabaceae & Platymiscium pintum & $\mathbb{F}$ & $U$ & 2 & - & - & - & - & - & - & - & - & - & - & - & - & - & - & - & - & - & - & & - \\
\hline Fabaceae & Platypodium elegans & $\mathbb{F}$ & $D$ & - & - & - & - & - & - & - & - & - & 1 & - & - & - & - & - & - & - & - & - & & - \\
\hline Fabaceae & $\begin{array}{l}\text { Poecilanthe effusa } \\
\text { Pseudopiptadenia }\end{array}$ & $\mathbb{F}$ & $U$ & - & - & - & - & - & 1 & - & - & - & - & - & - & - & - & - & - & - & - & - & & - \\
\hline Fabaceae & psilostachya & $\mathbb{F}$ & $U$ & - & - & - & - & - & 1 & - & - & - & - & - & - & - & - & - & - & - & - & - & & _- \\
\hline
\end{tabular}




\begin{tabular}{|c|c|c|c|c|c|c|c|c|c|c|c|c|c|c|c|c|c|c|c|c|c|c|c|}
\hline Family & Species & $\mathbb{V}$ & In & 它 & 㝴 & ف돌 & ') & 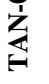 & 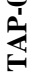 & $\stackrel{\Xi}{\leftrightarrows}$ & $\bigcup^{\circlearrowright}$ & & 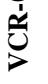 & 这 & 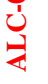 & 空 & 齐 & 齐 & 它 & 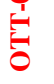 & $\sum_{n}$ & $\sum_{n=5}^{5}$ & $\sum_{\infty}^{E}$ \\
\hline Fabaceae & Pterodon emarginatus & $\mathfrak{S}$ & $D$ & _- & - & - & - & - & - & - & - & - & - & - & - & 1 & - & - & - & - & - & - & - \\
\hline Fabaceae & Pterodon pubescens & $\mathbb{S}$ & $U$ & - & - & - & - & - & - & - & - & - & - & - & - & - & - & - & - & - & - & 2 & - \\
\hline Fabaceae & Samanea tubulosa & $U$ & $D$ & - & - & - & - & - & - & 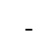 & - & . & - & - & 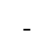 & . & 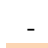 & - & - & 1 & 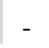 & 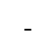 & - \\
\hline Fabaceae & $\begin{array}{l}\text { Sclerolobium aureum } \\
\text { Sclerolobium }\end{array}$ & $\mathfrak{S}$ & $S D$ & - & - & - & - & - & - & - & - & - & - & - & 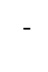 & - & 2 & - & - & - & 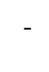 & - & 2 \\
\hline Fabaceae & chrysophyllum & IF & $U$ & - & - & - & - & - & 7 & 2 & - & - & - & - & - & $\ldots$ & - & - & - & - & - & _ & - \\
\hline Fabaceae & $\begin{array}{l}\text { Sclerolobium guianense } \\
\text { Sclerolobium }\end{array}$ & IF & $U$ & 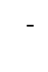 & - & - & - & - & - & 1 & - & - & - & - & - & - & - & - & - & - & - & - & - \\
\hline Fabaceae & $\begin{array}{l}\text { paniculatum } \\
\text { Stryphnodendron }\end{array}$ & $\mathfrak{S}$ & $U$ & - & - & - & - & - & - & - & - & - & 3 & - & 1 & - & - & 1 & - & - & - & - & - \\
\hline Fabaceae & $\begin{array}{l}\text { adstringens } \\
\text { Stryphnodendron }\end{array}$ & $\mathfrak{S}$ & $B D$ & - & - & - & - & - & - & - & - & - & - & - & - & - & - & - & - & - & - & - & - \\
\hline Fabaceae & $\begin{array}{l}\text { fissuratum } \\
\text { Stryphnodendron }\end{array}$ & IF & $S D$ & - & - & - & - & - & - & - & - & - & - & - & - & - & - & - & 1 & - & - & - & - \\
\hline Fabaceae & obovatum & $\mathfrak{S}$ & $U$ & - & - & - & - & - & - & - & - & - & - & - & - & - & - & - & 2 & - & - & - & - \\
\hline Fabaceae & Tachigali myrmecophila & IF & $U$ & - & - & - & - & - & 1 & 1 & - & - & - & - & - & - & - & - & - & - & - & - & - \\
\hline Fabaceae & Vatairea macrocarpa & $\mathfrak{S}$ & $D$ & - & - & - & - & - & - & - & - & - & - & - & - & - & - & - & - & - & - & 2 & 1 \\
\hline Flacourtiaceae & Bara guianensis & $\mathfrak{S}$ & $U$ & - & - & - & - & - & - & - & - & - & - & - & 1 & - & - & - & - & - & - & - & - \\
\hline Humiriaceae & Sacoglottis guianensis & IF & $S D$ & - & 2 & - & - & 1 & - & - & - & - & - & - & - & - & - & - & - & - & - & - & - \\
\hline Icaciceae & Emmotum nitens & $\mathfrak{S}$ & $E$ & - & - & - & - & - & - & - & - & - & - & - & - & - & - & 5 & - & - & 5 & 3 & 1 \\
\hline Lauraceae & Mezilaurus crassiramea & $\mathfrak{S}$ & $B D$ & - & - & - & - & - & - & - & - & - & - & - & - & - & 1 & 1 & - & - & . & 3 & 2 \\
\hline Lauraceae & Mezilaurus lindavia & IF & $U$ & - & - & - & - & - & - & 1 & - & - & - & - & - & - & - & - & - & - & - & - & - \\
\hline Lauraceae & Nectandra cuspidata & $\mathbb{F}$ & $S D$ & - & - & - & - & 1 & - & - & - & - & 1 & - & - & - & - & - & - & - & - & - & - \\
\hline Lauraceae & Nectandra hihua & IF & $S D$ & - & - & - & - & - & - & - & - & - & 2 & - & - & - & - & - & - & - & - & - & - \\
\hline Lauraceae & Nectandra sp. & $U$ & $U$ & - & 1 & - & - & - & - & - & - & - & - & - & - & - & - & - & - & - & - & - & - \\
\hline Lauraceae & Ocotea guianensis & IF & $U$ & - & 3 & - & - & 3 & - & - & - & - & - & - & - & - & - & - & - & - & - & - & - \\
\hline Lauraceae & Ocotea hoehnei & IF & $U$ & - & - & - & - & - & - & - & - & - & 1 & - & - & - & - & - & - & - & - & - & - \\
\hline Lauraceae & Ocotea leucoxylon & IF & $U$ & - & 2 & - & - & 3 & - & - & - & - & - & - & - & - & - & - & - & - & - & - & - \\
\hline Lecythidaceae & Eschweilera albiflora & IF & $U$ & - & - & - & - & - & - & - & - & - & - & - & - & - & - & - & - & - & - & - & - \\
\hline Lecythidaceae & Eschweilera blanchetia & IF & $U$ & - & - & - & - & - & - & 1 & 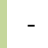 & - & - & - & - & - & - & - & - & - & - & - & - \\
\hline Lecythidaceae & Eschweilera odora & $\mathbb{F}$ & $U$ & - & - & - & - & - & - & 1 & - & - & - & - & - & - & - & - & - & - & - & - & - \\
\hline Lecythidaceae & Lecythis lurida & IF & $U$ & - & - & - & - & - & 8 & - & - & - & - & - & - & - & - & - & - & - & - & - & - \\
\hline Loganiaceae & Antonia ovata & $\mathfrak{S}$ & $U$ & - & - & - & - & - & - & - & - & - & - & - & - & - & 1 & - & - & - & - & - & - \\
\hline Loganiaceae & Strychnos pseudoquina & $\mathfrak{S}$ & $U$ & - & - & - & - & - & - & - & - & - & - & - & - & - & 3 & 1 & - & - & - & - & 1 \\
\hline Lythraceae & Lafoensia pacari & $\mathfrak{S}$ & $D$ & - & - & - & - & - & - & - & - & - & - & - & 4 & - & 1 & - & - & - & 2 & - & - \\
\hline Malpighiaceae & Banisteriopsis pubipetala & $\mathfrak{S}$ & $U$ & - & - & - & - & - & - & - & - & - & - & - & - & - & - & 1 & - & - & - & - & - \\
\hline Malpighiaceae & Byrsonima basiloba & $\mathfrak{S}$ & $S D$ & - & - & - & - & - & - & - & - & - & - & - & - & - & - & - & - & - & - & 1 & - \\
\hline Malpighiaceae & $\begin{array}{l}\text { Byrsonima chrysophylla } \\
\text { Byrsonima }\end{array}$ & $\mathfrak{S}$ & $U$ & - & - & - & - & - & - & - & - & - & - & 7 & 4 & - & - & - & - & - & - & - & - \\
\hline Malpighiaceae & coccolobaefolia & $\mathfrak{S}$ & $B D$ & - & - & - & - & - & - & - & - & - & - & 4 & 2 & - & 1 & 1 & - & - & - & 1 & - \\
\hline Malpighiaceae & Byrsonima crassa & $\mathfrak{S}$ & $U$ & - & - & - & - & - & - & - & - & - & - & - & - & - & - & - & - & - & 1 & - & - \\
\hline Malpighiaceae & Byrsonima fagifolia & $\mathfrak{S}$ & $U$ & - & - & - & - & - & - & - & - & - & - & - & - & 2 & - & - & - & - & - & - & - \\
\hline Malpighiaceae & Byrsonima intermedia & $\mathfrak{S}$ & $U$ & - & 1 & - & - & - & - & - & - & - & - & - & - & - & - & - & - & - & - & - & - \\
\hline Malpighiaceae & Byrsonima pachyphylla & $\mathfrak{S}$ & $U$ & - & - & - & - & - & - & - & - & - & - & - & - & - & - & - & - & - & 1 & - & 4 \\
\hline Malpighiaceae & Byrsonima verbascifolia & $\mathfrak{S}$ & $S D$ & - & - & - & - & - & - & - & - & - & - & - & - & - & - & - & - & - & - & 1 & 1 \\
\hline Malpighiaceae & Heteropterys eglandulosa & IF & $U$ & - & - & - & - & - & - & - & - & 1 & - & - & - & - & - & - & - & - & - & - & - \\
\hline Malpighiaceae & Licania apetala & $\mathbb{F}$ & $U$ & - & - & - & - & - & - & - & - & - & 1 & - & - & - & - & - & - & - & - & - & - \\
\hline Malpighiaceae & Licania blackii & $\mathbb{F}$ & $U$ & - & 2 & - & - & - & - & - & - & - & - & - & - & - & - & - & - & - & - & - & - \\
\hline Malpighiaceae & Licania gardneri & IF & $U$ & - & 1 & - & - & - & - & - & - & - & - & - & - & - & - & - & - & - & - & - & - \\
\hline Malpighiaceae & Licania heteromorpha & IF & $U$ & - & - & - & - & - & - & 1 & - & - & - & - & - & - & - & - & - & - & - & - & - \\
\hline Malpighiaceae & Licania humilis & $\mathfrak{S}$ & $U$ & - & - & - & - & - & - & - & - & - & - & - & - & - & - & - & - & - & - & - & 1 \\
\hline Malpighiaceae & Licania minutiflora & $\mathbb{F}$ & $U$ & 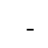 & 5 & - & - & 2 & - & - & - & - & - & - & - & - & - & - & - & - & - & - & - \\
\hline
\end{tabular}




\begin{tabular}{|c|c|c|c|c|c|c|c|c|c|c|c|c|c|c|c|c|c|c|c|c|c|c|c|c|c|c|}
\hline Family & Species & 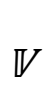 & In & 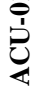 & ơ & 㝘 & ס & 竞 & 品 & 光 & فَ & 齐 & 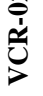 & Uِ & נִ & & & & & & 5 & 㿠 & $\sum_{\infty}^{i}$ & & & \\
\hline Malvaceae & Ceiba insignis & $\mathbb{F}$ & $D$ & - & - & - & - & - & - & - & 3 & - & - & & 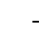 & & & - & & & 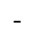 & - & - & & - & - \\
\hline Malvaceae & Ceiba speciosa & $\mathbb{F}$ & $D$ & - & - & - & 3 & - & - & - & - & - & - & - & - & & & - & & & - & - & - & & - & - \\
\hline Malvaceae & Eriotheca gracilipes & $\mathcal{S}$ & $B D$ & - & - & - & - & - & - & - & - & - & - & _ & - & & & 3 & & & - & 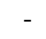 & - & & 1 & 3 \\
\hline Malvaceae & Eriotheca pubescens & $\mathcal{S}$ & $S D$ & - & - & - & - & - & - & - & - & - & - & 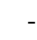 & - & & - & - & & & 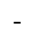 & 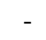 & - & & - & - \\
\hline Malvaceae & Eriotheca roseorum & $\mathbb{F}$ & $U$ & 1 & - & - & - & - & - & - & - & - & - & - & - & & & - & & & - & - & - & & - & - \\
\hline Malvaceae & Erisma uncitum & $\mathbb{F}$ & $U$ & - & - & 4 & - & - & - & - & - & - & - & - & - & & & - & & & - & - & - & & - & - \\
\hline Malvaceae & Guazuma ulmifolia & $\mathbb{F}$ & $B D$ & - & - & - & - & - & - & - & - & - & - & - & - & & & - & & & - & 1 & - & & - & - \\
\hline Malvaceae & Luehea paniculata & $\mathcal{S}$ & $U$ & - & - & - & - & - & - & - & - & - & - & - & - & & & & & & . & 1 & - & & - & - \\
\hline Malvaceae & Luehea tomentella & $\mathcal{S}$ & $D$ & - & - & - & - & - & - & - & - & - & - & - & - & & & & & & - & 1 & - & & - & - \\
\hline $\begin{array}{l}\text { Malvaceae } \\
\text { Melastomatacea }\end{array}$ & Lueheopsis duckea & $\mathbb{F}$ & $U$ & - & - & - & - & - & - & - & - & - & - & - & - & & - & - & & & - & - & - & & - & - \\
\hline $\begin{array}{l}\text { e } \\
\text { Melastomatacea }\end{array}$ & Bellucia dichotoma & $\mathbb{F}$ & $U$ & - & - & 1 & - & - & - & - & - & - & - & - & - & & - & 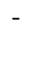 & & & - & - & - & & - & - \\
\hline $\begin{array}{l}\text { e } \\
\text { Melastomatacea }\end{array}$ & Bellucia grossularioides & $\mathbb{F}$ & $U$ & - & 2 & - & - & 1 & - & - & - & - & - & - & - & & - & - & & & - & - & - & & - & - \\
\hline $\begin{array}{l}\text { e } \\
\text { Melastomatacea }\end{array}$ & Miconia albicans & $\mathcal{S}$ & $E$ & - & - & - & - & - & - & - & - & - & - & - & 3 & & 1 & - & & & - & - & 1 & & - & - \\
\hline $\begin{array}{l}\text { e } \\
\text { Melastomatacea }\end{array}$ & Miconia chrysophylla & $\mathbb{F}$ & $E$ & - & - & 1 & - & - & - & - & - & - & - & - & - & & - & - & & & - & - & - & & - & - \\
\hline $\begin{array}{l}\text { e } \\
\text { Melastomatacea }\end{array}$ & Miconia cuspidata & $\mathbb{F}$ & $E$ & - & 1 & - & - & - & - & - & - & - & - & - & - & & - & - & & & - & - & - & & - & - \\
\hline $\begin{array}{l}\text { e } \\
\text { Melastomatacea }\end{array}$ & Miconia ferrugita & $\mathcal{S}$ & $E$ & - & - & - & - & - & - & - & - & - & - & - & - & & - & - & & & - & - & - & & - & - \\
\hline $\begin{array}{l}\text { e } \\
\text { Melastomatacea }\end{array}$ & Miconia indet & $U$ & $U$ & - & - & 1 & - & - & - & - & - & - & - & - & - & & - & - & & & - & - & - & & - & - \\
\hline $\begin{array}{l}\text { e } \\
\text { Melastomatacea }\end{array}$ & Miconia lepidota & $\mathbb{F}$ & $U$ & - & - & - & - & - & 1 & - & - & - & - & - & - & & - & - & & & - & - & - & & - & - \\
\hline $\begin{array}{l}\text { e } \\
\text { Melastomatacea }\end{array}$ & Miconia pyrifolia & $\mathbb{F}$ & $E$ & - & 1 & - & - & 6 & - & - & - & - & - & - & - & & - & - & & & - & - & - & & - & - \\
\hline $\begin{array}{l}\text { e } \\
\text { Melastomatacea }\end{array}$ & Miconia tomentosa & $\mathbb{F}$ & $E$ & - & 1 & - & - & - & - & - & - & - & - & - & - & & - & - & & & - & - & - & & - & - \\
\hline $\begin{array}{l}\text { e } \\
\text { Melastomatacea }\end{array}$ & Mouriri brachyanthera & $\mathbb{F}$ & $U$ & - & - & - & - & 3 & 1 & - & - & - & - & - & - & & - & - & & & - & - & - & & - & - \\
\hline $\mathrm{e}$ & Mouriri elliptica & $\mathcal{S}$ & $S D$ & - & - & - & - & - & - & - & - & - & - & - & - & & & 2 & & & - & - & 4 & & 1 & 1 \\
\hline Meliaceae & Guarea kunthiana & $\mathbb{F}$ & $S D$ & - & - & - & - & - & - & - & - & - & - & - & - & & & - & & & - & - & - & & - & - \\
\hline Meliaceae & Trichilia micrantha & $\mathbb{F}$ & $U$ & - & 1 & - & - & - & - & - & - & - & - & - & - & & & - & & & - & - & - & & - & - \\
\hline Meliaceae & Trichilia quadrijuga & $\mathbb{F}$ & $U$ & - & - & - & - & - & - & - & - & - & - & - & - & & - & - & & & - & - & - & & - & - \\
\hline Meliaceae & Trichilia sp. & $U$ & $U$ & - & 1 & - & - & - & - & - & - & - & - & - & - & & & - & & & - & - & - & & - & - \\
\hline Monimiaceae & Siparuna decipiens & $\mathbb{F}$ & $U$ & - & - & - & - & - & 1 & - & - & - & - & - & - & & - & - & & & - & - & - & & 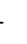 & - \\
\hline Moraceae & Brosimum gaudichaudii & $\mathcal{S}$ & $U$ & - & - & - & - & - & - & - & - & - & - & - & - & & & - & & & - & 1 & - & & - & - \\
\hline Moraceae & Brosimum paririoides & $\mathbb{F}$ & $U$ & - & - & - & - & - & 1 & - & - & $\begin{array}{l}- \\
1\end{array}$ & - & - & - & & - & - & & & - & - & - & & - & - \\
\hline Moraceae & Brosimum rubescens & $\mathbb{F}$ & $S D$ & - & - & - & - & - & - & - & - & 2 & 5 & - & - & & & - & & & - & - & - & & - & - \\
\hline Moraceae & Helicostylis pedunculata & $\mathbb{F}$ & $U$ & - & - & - & - & - & 1 & - & - & - & - & - & - & & & - & & & - & - & - & & - & - \\
\hline Moraceae & Maclura tinctoria & $\mathbb{F}$ & $D$ & - & - & - & 1 & - & - & - & - & - & - & - & - & & & - & & & - & - & - & & - & - \\
\hline Moraceae & Maquira guianensis & $\mathcal{S}$ & $D$ & - & - & - & - & - & 1 & - & - & - & - & - & - & & - & - & & & - & - & - & & - & - \\
\hline Moraceae & Maquira sclerophylla & $\mathbb{F}$ & $U$ & - & - & - & - & - & - & 1 & - & - & - & - & - & & - & - & & & - & - & - & & 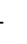 & - \\
\hline Moraceae & Perebea mollis & $\mathbb{F}$ & $U$ & - & - & - & - & - & 1 & - & - & - & - & - & - & & - & - & & & - & - & - & & - & - \\
\hline Moraceae & Pseudolmedia laevigata & $\mathbb{F}$ & $U$ & - & 2 & - & - & 4 & - & - & - & - & - & - & - & & - & - & & & - & - & - & & - & - \\
\hline Moraceae & $\begin{array}{l}\text { Pseudolmedia laevis } \\
\text { Pseudolmedia }\end{array}$ & $\mathbb{F}$ & $U$ & - & - & 3 & - & - & - & - & - & - & - & - & - & & - & - & & & - & - & - & & - & - \\
\hline Moraceae & macrophylla & $\mathbb{F}$ & $U$ & - & - & 4 & - & - & - & - & - & - & - & - & - & & - & - & & & - & - & - & & - & - \\
\hline Moraceae & Sahagunia racemifera & $\mathbb{F}$ & $U$ & - & - & - & - & - & - & 2 & - & - & - & - & - & & & - & & & - & - & - & & - & - \\
\hline Myristicaceae & Iryanthera laevis & $\mathbb{F}$ & $U$ & - & - & 1 & - & - & - & - & - & - & - & - & - & & & - & & & - & - & - & & - & - \\
\hline Myristicaceae & Virola michelii & $\mathbb{F}$ & $U$ & - & - & - & - & - & 1 & - & - & - & - & - & - & & & - & & & - & - & - & & - & - \\
\hline Myristicaceae & Virola sebifera & $\mathbb{F}$ & $U$ & - & - & - & - & - & - & - & - & - & 1 & - & - & & & - & & & - & - & - & & - & - \\
\hline Myrtaceae & Blepharocalyx salicifolius & $\mathcal{S}$ & $B D$ & - & - & - & - & - & - & - & - & - & - & - & - & & - & 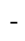 & & & - & - & - & & - & - \\
\hline
\end{tabular}




\begin{tabular}{|c|c|c|c|c|c|c|c|c|c|c|c|c|c|c|c|c|c|c|c|c|c|c|c|c|}
\hline Family & Species & $V$ & In & 总 & & 这 & 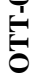 & 离 & 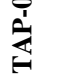 & 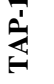 & 己ِ & ত্் & 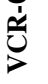 & U్ & נִ & 皇 & 纭 & 穵 & ') & : & & & & $\sum_{i}$ \\
\hline Myrtaceae & Eugenia dysenterica & $\mathcal{S}$ & $D$ & - & - & - & - & - & - & - & - & - & - & - & - & 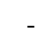 & 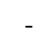 & - & - & - & & & & 3 \\
\hline Myrtaceae & Eugenia gemmiflora & $\mathcal{S}$ & $U$ & - & - & - & - & - & - & - & - & - & - & - & - & 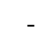 & . & - & - & - & & & & 2 \\
\hline Myrtaceae & Eugenia omissa & IF & $U$ & - & - & - & - & - & 1 & - & - & - & - & - & - & - & - & - & - & - & . & & & - \\
\hline Myrtaceae & Myrcia multiflora & $\mathbb{S}$ & $U$ & - & - & - & - & - & - & - & - & - & - & - & - & _ & - & - & - & - & 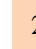 & & & - \\
\hline Myrtaceae & Myrcia sellowiana & $\mathbb{S}$ & $E$ & - & - & - & - & - & - & - & - & - & - & - & - & . & - & 4 & - & - & 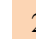 & 2 & & - \\
\hline Myrtaceae & Psidium sartorianum & F & $U$ & 4 & - & - & - & - & - & - & - & - & - & - & - & - & - & - & - & - & & & & - \\
\hline Not known & Unidentified & $U$ & $U$ & - & - & - & - & - & - & - & - & - & - & - & - & 1 & 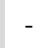 & - & - & - & & & & - \\
\hline Not known & Unidentified & $U$ & $U$ & - & - & - & - & - & - & - & - & - & - & - & - & 1 & - & - & - & - & . & & & - \\
\hline Not known & Unidentified & $U$ & $U$ & - & - & - & 1 & - & - & - & - & - & - & - & - & - & - & - & - & - & & & & - \\
\hline Not known & Unidentified & $U$ & $U$ & - & - & - & - & - & - & - & - & - & - & 1 & - & - & - & - & - & - & & & - & - \\
\hline Not known & Unidentified & $U$ & $U$ & - & - & - & - & - & - & - & - & - & - & 1 & - & - & - & - & - & - & & & - & - \\
\hline Not known & Unidentified & $U$ & $U$ & - & - & - & - & - & - & - & - & - & - & 1 & - & - & - & - & - & - & & & - & - \\
\hline Not known & Unidentified & $U$ & $U$ & - & - & - & - & 1 & - & - & - & - & - & - & - & - & - & - & - & - & & & - & - \\
\hline Nyctagiceae & Guapira graciliflora & $\mathbb{S}$ & $D$ & - & - & - & - & - & - & - & - & - & - & - & - & - & - & 2 & - & - & & & 1 & - \\
\hline Nyctagiceae & Guapira noxia & $\mathcal{S}$ & $D$ & - & - & - & - & - & - & - & - & - & - & - & - & - & 2 & - & - & - & & & - & - \\
\hline Nyctagiceae & Neea hermaphrodita & F & $U$ & - & - & - & - & - & - & - & 3 & - & - & - & - & - & - & - & - & - & & & - & - \\
\hline Nyctagiceae & Neea sprucea & IF & $U$ & - & - & - & - & - & - & - & - & - & - & - & - & - & - & - & - & - & & & - & - \\
\hline Nyctagiceae & Pisonia zapallo & F & $U$ & - & - & - & - & - & - & - & 1 & - & - & - & - & - & - & - & - & - & & & - & - \\
\hline Ochnaceae & Ouratea discophora & F & $U$ & - & 2 & - & - & - & - & - & - & - & - & - & - & - & - & - & - & - & . & & - & - \\
\hline Ochnaceae & Ouratea hexasperma & $\mathbb{S}$ & $U$ & - & - & - & - & - & - & - & - & - & - & - & - & - & - & - & - & - & 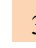 & & - & - \\
\hline Ochnaceae & Ouratea spectabilis & $\mathbb{S}$ & $U$ & - & - & - & - & - & - & - & - & - & - & - & - & 1 & 1 & - & - & - & & & - & - \\
\hline Olacaceae & $\begin{array}{l}\text { Minquartia guianensis } \\
\text { Priogymnthus }\end{array}$ & IF & $U$ & - & - & - & - & - & - & 1 & - & - & - & - & - & - & - & - & - & - & & & - & - \\
\hline Oleaceae & hasslerianus & $\mathfrak{S}$ & $D$ & - & - & - & - & - & - & - & - & - & - & - & - & - & - & - & - & - & & & 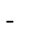 & - \\
\hline Opiliaceae & Agonandra brasiliensis & $\mathcal{S}$ & $U$ & - & - & - & - & - & - & - & - & - & - & - & - & - & - & 1 & - & - & & & - & - \\
\hline Polygonaceae & Coccoloba mollis & $\mathcal{S}$ & $U$ & - & - & - & - & - & - & - & - & - & - & - & - & - & - & 1 & - & - & & & - & - \\
\hline Polygonaceae & Ruprechtia triflora & F & $D$ & - & - & - & - & - & - & - & 1 & - & - & - & - & - & - & - & - & - & & & - & - \\
\hline Proteaceae & Euplassa inaequalis & $\mathfrak{S}$ & $S D$ & - & - & - & - & - & - & - & - & - & - & - & - & - & 5 & - & - & - & . & & 2 & - \\
\hline Proteaceae & $\begin{array}{l}\text { Roupala montana } \\
\text { Rhamnidium }\end{array}$ & $\mathcal{S}$ & $S D$ & - & - & - & - & - & - & - & - & - & - & 1 & - & 1 & 1 & 1 & - & - & 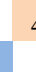 & & 2 & 2 \\
\hline Rhamnaceae & elaeocarpum & $\mathbb{F}$ & $B D$ & - & - & - & - & - & - & - & - & - & - & - & - & - & - & - & - & 1 & 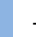 & & & - \\
\hline Rubiaceae & $\begin{array}{l}\text { Amaioua guianensis } \\
\text { Calycophyllum }\end{array}$ & $\mathbb{F}$ & E & - & 5 & - & - & 2 & - & - & - & 1 & 5 & - & - & - & - & - & - & - & & & - & - \\
\hline Rubiaceae & multiflorum & FF & $B D$ & - & - & - & 1 & - & - & - & - & - & - & - & - & - & - & - & - & - & & & - & - \\
\hline Rubiaceae & Coussarea racemosa & F & $U$ & - & - & - & - & - & 3 & - & - & - & - & - & - & - & - & - & - & - & & & - & - \\
\hline Rubiaceae & Ferdinandusa elliptica & $\mathfrak{S}$ & $U$ & - & - & - & - & - & - & - & - & - & - & - & - & 1 & - & - & - & - & & & - & - \\
\hline Rubiaceae & Ferdinandusa rudgeoides & $\mathbb{F}$ & $U$ & - & - & - & - & 2 & - & - & - & - & - & - & - & - & - & - & - & - & 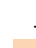 & & - & - \\
\hline Rubiaceae & Tocoyena formosa & $\mathcal{S}$ & $U$ & - & - & - & - & - & - & - & - & - & - & - & - & - & - & - & - & - & 2 & & - & - \\
\hline Salicaceae & Casearia arborea & $U$ & $E$ & - & 1 & - & - & - & - & - & - & - & - & - & - & - & - & - & - & - & & & - & - \\
\hline Salicaceae & Casearia sylvestris & $\mathcal{S}$ & $U$ & - & - & - & - & - & - & - & - & - & - & - & - & - & - & - & - & - & & & - & 1 \\
\hline Sapindaceae & Astronium urundeuva & $\mathfrak{S}$ & $U$ & - & - & - & - & - & - & - & - & - & - & - & - & - & - & - & - & - & 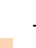 & & - & - \\
\hline Sapindaceae & Dilodendron bipinatum & $\mathfrak{S}$ & $D$ & 1 & - & - & - & - & - & - & - & - & - & - & - & - & - & - & - & 3 & . & & - & - \\
\hline Sapindaceae & Magonia pubescens & $\mathcal{S}$ & $D$ & - & - & - & - & - & - & - & - & - & - & - & - & - & 1 & - & 7 & 6 & 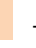 & & - & - \\
\hline Sapindaceae & Matayba guianensis & $\mathcal{S}$ & $D$ & - & - & - & - & 4 & - & - & - & - & 1 & - & - & - & - & 2 & - & - & & & - & - \\
\hline Sapindaceae & Talisia longifolia & $\mathbb{F}$ & $U$ & - & - & - & - & - & - & 1 & - & - & - & - & - & - & - & - & - & - & & & - & - \\
\hline Sapindaceae & Talisia sp. & $U$ & $U$ & - & - & - & - & 2 & - & - & - & - & - & - & - & - & - & - & - & - & & & - & - \\
\hline Sapotaceae & Ecclinusa ramiflora & IF & $U$ & - & - & - & - & - & $\begin{array}{l}1 \\
1\end{array}$ & - & - & - & - & - & - & - & - & - & - & - & & & & - \\
\hline Sapotaceae & Manilkara huberi & $\mathbb{F}$ & $U$ & - & - & - & - & - & 1 & 1 & - & - & - & - & - & - & - & - & - & - & & & - & - \\
\hline Sapotaceae & Micropholis egensis & F & $U$ & - & - & 1 & - & - & - & - & - & - & - & - & - & - & - & - & - & - & 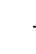 & - & - & - \\
\hline
\end{tabular}




\begin{tabular}{|c|c|c|c|c|c|c|c|c|c|c|c|c|c|c|c|c|c|c|c|c|c|c|c|c|c|c|c|}
\hline Family & Species & $V$ & In & 家 & 㝘 & 富 & $\begin{array}{l}0 \\
0 \\
0 \\
0\end{array}$ & 志 & 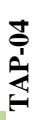 & 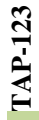 & $\begin{array}{l}\bar{\theta} \\
\dot{\sigma} \\
\underline{\sigma}\end{array}$ & $\bar{e}$ & & 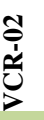 & 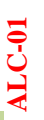 & 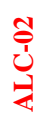 & $\frac{1}{1}$ & & $\begin{array}{l}\overline{0} \\
\overline{1} \\
z \\
z\end{array}$ & 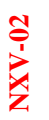 & ố & '1 & & & $\sum_{\mathscr{S}}^{\stackrel{N}{\sigma}}$ & & \\
\hline Sapotaceae & Micropholis venulosa & $\mathbb{F}$ & $U$ & - & 4 & - & - & 1 & - & 1 & . & & - & 1 & - & - & & 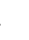 & - & - & - & & & - & L & & - \\
\hline Sapotaceae & Pouteria caimito & $\mathbb{F}$ & $U$ & - & - & - & - & - & 1 & - & - & & - & - & - & - & & . & - & - & - & & & - & - & & - \\
\hline Sapotaceae & Pouteria ramiflora & $\mathcal{S}$ & $B D$ & - & 1 & - & - & - & - & - & - & & - & - & - & - & & 1 & 1 & - & - & & & 1 & 3 & & 3 \\
\hline Sapotaceae & Pouteria sp. & $U$ & $U$ & - & - & - & - & 1 & - & - & - & & - & - & - & - & & & - & - & - & & & - & - & & - \\
\hline Sapotaceae & Pouteria torta & $\mathbb{F}$ & $S D$ & - & - & - & - & - & - & - & - & & - & 1 & - & - & & & - & - & - & & & - & - & & - \\
\hline Schoepfiaceae & Schoepfia obliquifolia & $\mathbb{F}$ & E & - & 1 & - & - & - & - & - & - & & - & - & - & - & & & - & - & - & & & - & - & & - \\
\hline Simaroubaceae & Simaba cedron & $\mathbb{F}$ & $U$ & - & - & - & - & - & - & 1 & - & & - & - & - & - & & . & - & - & - & & & - & - & & - \\
\hline Simaroubaceae & Simarouba amara & $\mathfrak{S}$ & $S D$ & - & - & - & - & - & - & - & - & & - & - & - & 2 & & . & - & - & - & & & - & - & & - \\
\hline Styracaceae & Styrax ferrugineus & $\mathcal{S}$ & $S D$ & - & - & - & - & - & - & - & 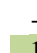 & & - & - & - & - & & & - & - & - & & & - & - & & - \\
\hline Urticaceae & Pourouma guianensis & $\mathbb{F}$ & $U$ & - & - & - & - & - & - & - & - & & - & - & - & - & & . & - & - & - & & & - & - & & - \\
\hline Verbeceae & Vitex triflora & $\mathbb{F}$ & $U$ & - & - & - & - & - & - & 1 & - & & - & - & - & - & & . & - & - & - & & & - & - & & - \\
\hline Violaceae & Rinorea guianensis & $\mathbb{F}$ & $U$ & - & - & - & - & - & - & 1 & - & & - & - & - & - & & . & - & - & - & & & - & - & & - \\
\hline Violaceae & Rinoreocarpus ulei & $\mathbb{F}$ & $U$ & - & - & - & - & - & - & - & & & - & - & - & - & & & - & - & - & & & - & - & & - \\
\hline Vochysiaceae & Qualea grandiflora & $\mathcal{S}$ & $D$ & - & - & - & - & - & - & - & - & & - & - & 1 & 4 & & . & 2 & - & - & & & 1 & 2 & & - \\
\hline Vochysiaceae & Qualea multiflora & $S$ & $U$ & - & - & - & - & - & - & - & - & & - & - & - & - & & 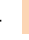 & 1 & - & - & & & 1 & 2 & & - \\
\hline Vochysiaceae & Qualea paraensis & $\mathbb{F}$ & $U$ & - & - & 5 & - & - & - & - & & & - & - & - & - & & . & - & - & - & & & - & - & & - \\
\hline Vochysiaceae & Qualea parviflora & $\mathfrak{S}$ & $U$ & - & - & - & - & - & - & - & . & & - & - & - & - & & . & 2 & - & - & & & - & 1 & & - \\
\hline Vochysiaceae & Qualea spp. & $U$ & $U$ & - & - & - & - & - & - & - & & & - & - & 2 & 2 & & . & - & - & - & & & - & - & & - \\
\hline Vochysiaceae & Salvertia convallariodora & $\mathcal{S}$ & $S D$ & - & - & - & - & - & - & 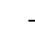 & . & & - & - & 6 & 4 & & 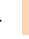 & 1 & - & - & & & - & - & & 1 \\
\hline Vochysiaceae & Vochysia haenkeana & $\mathcal{S}$ & $U$ & - & - & - & - & - & - & . & - & & - & - & - & - & & . & - & - & - & & & - & 1 & & - \\
\hline Vochysiaceae & Vochysia thyrsoidea & $\mathcal{S}$ & $U$ & - & - & - & - & - & - & - & & & - & - & - & - & & & - & - & - & & & - & - & & - \\
\hline Vochysiaceae & Vochysia vismiifolia & $\mathbb{F}$ & $U$ & - & 1 & - & - & 1 & - & - & & & - & - & - & - & & . & - & - & - & & & - & - & & - \\
\hline
\end{tabular}

Table S2. List of measurement species along with their primary vegetation affiliation ( $\mathscr{V}$ ), leaf habit (Ih) and number of samples taken per plot. $\mathbb{F}=$ primarily forest affiliated, = primarily savanna affiliated; $B D=$ brevideciduous, $D=$ deciduous, $E=$ evergreen, $S D=$ semi-deciduous, $U=$ unknown. Where sampled in the same plot as their primary affiliation, forest trees cells are coloured green and savanna trees coloured light brown. Where sampled in the other $s$ to which they usually occur, forest trees are coloured blue and savanna trees are coloured maroon. Trees not identified to the species level are cell coloured grey with this also being the case for a few species for which $\mathbb{V}$ ) was not known equivocally. 


\section{Fixed Effect}

\begin{tabular}{|c|c|c|}
\hline Parametric terms & Coefficient & S.E. \\
\hline$\mu=$ intercept: $\mathbb{F}$ dataset mean, $\langle\mathbb{F}\rangle$ & 115.1 & 4.9 \\
\hline$\alpha=$ savanna - forest difference: $\langle\mathcal{S}\rangle-\langle\mathbb{F}\rangle$ & 25.6 & 5.7 \\
\hline Random effect & \multicolumn{2}{|c|}{ Variance component } \\
\hline Level 2 variances & & \\
\hline Plot & \multicolumn{2}{|c|}{295.0} \\
\hline Species & \multicolumn{2}{|c|}{108.1} \\
\hline Level 1 variance & & \\
\hline Residual & \multicolumn{2}{|c|}{1498.6} \\
\hline
\end{tabular}

Table S3a: Mixed model predictions for leaf mass per unit area $\left(M_{\mathrm{a}}: \mathrm{g} \mathrm{m}^{-2}\right)$ as in Eqn 4, but with species affiliation (बl) treated as a fixed rather than a random effect.

\section{Fixed Effect}

\begin{tabular}{|c|c|c|c|}
\hline Parametric terms & Coefficient & S.E. & $t$ \\
\hline$\mu=$ intercept: $\mathbb{F}$ dataset mean, $\langle\mathbb{F}\rangle$ & 21.20 & 1.05 & 20.14 \\
\hline$\alpha=$ savanna - forest difference: $\langle\mathscr{S}\rangle-\langle\mathbb{F}\rangle$ & -2.87 & 1.08 & -2.66 \\
\hline Random effect & \multicolumn{2}{|c|}{ Variance component } & \\
\hline Level 2 variances & & & \\
\hline Plot & \multicolumn{2}{|c|}{16.06} & \\
\hline Species & \multirow{2}{*}{\multicolumn{2}{|c|}{9.23}} & \\
\hline Level 1 variance & & & \\
\hline Residual & \multicolumn{2}{|c|}{25.02} & \\
\hline
\end{tabular}

Table S3b: Mixed model predictions for leaf nitrogen per unit dry mass $\left(N_{\mathrm{m}}: \mathrm{mg} \mathrm{g}^{-1}\right)$ as in Eqn 4, but with species affiliation (बl) treated as a fixed rather than a random effect.

\section{Fixed Effect}

\begin{tabular}{|c|c|c|c|}
\hline Parametric terms & Coefficient & S.E. & $t$ \\
\hline$\mu=$ intercept: $\mathbb{F}$ dataset mean, $\langle\mathbb{F}\rangle$, & 475.41 & 4.94 & 96.2 \\
\hline$\alpha=$ savanna - forest difference: $\langle S \mathcal{S}\rangle-\langle\mathbb{F}\rangle$ & 20.31 & 7.03 & 2.89 \\
\hline Random effect & \multicolumn{2}{|c|}{ Variance component } & \\
\hline Level 2 variances & & & \\
\hline Plot & \multicolumn{2}{|c|}{34.8} & \\
\hline Species & \multirow{2}{*}{\multicolumn{2}{|c|}{590.2}} & \\
\hline Level 1 variance & & & \\
\hline Residual & \multicolumn{2}{|c|}{4547.7} & \\
\hline
\end{tabular}

Table S3c: Mixed model predictions for leaf carbon per unit dry mass $\left(C_{\mathrm{m}}: \mathrm{mg} \mathrm{g}^{-1}\right)$ as in Eqn 4, but with species affiliation (बl) treated as a fixed rather than a random effect. 


\section{Fixed Effect}

\begin{tabular}{|c|c|c|}
\hline Parametric terms & Coefficient & S.E. \\
\hline$\mu=$ intercept: $\mathbb{F}$ dataset mean, $\langle\mathbb{F}\rangle$, & 0.809 & 0.043 \\
\hline$\alpha=$ savanna - forest difference: $\langle S \mathcal{S}\rangle-\langle\mathbb{F}\rangle$ & -0.036 & 0.049 \\
\hline Random effect & \multicolumn{2}{|c|}{ Variance component $\left(\mathrm{x}^{10^{3}}\right)$} \\
\hline Level 2 variances & & \\
\hline Plot & \multicolumn{2}{|c|}{21.6} \\
\hline Species & \multicolumn{2}{|c|}{2.8} \\
\hline Level 1 variance & & \\
\hline Residual & \multicolumn{2}{|c|}{133.7} \\
\hline
\end{tabular}

Table S3d: Mixed model predictions for leaf phosphorus per unit dry mass $\left(P_{\mathrm{m}}: \mathrm{mg} \mathrm{g}^{-1}\right)$ as in Eqn 4, but with species affiliation (बl) treated as a fixed rather than a random effect.

\section{Fixed Effect}

\begin{tabular}{lccc}
\hline Parametric terms & Coefficient & S.E. & $\boldsymbol{t}$ \\
\cline { 2 - 4 }$\mu=$ intercept: $\mathbb{F}$ dataset mean, $\langle\mathbb{F}\rangle$, & 8.54 & 0.88 & 9.73 \\
$\alpha=$ savanna - forest difference: $\langle\mathscr{S}\rangle-\langle\mathbb{F}\rangle$ & -2.90 & 0.99 & -2.92
\end{tabular}

\begin{tabular}{lc}
\hline Random effect & Variance component $\left(\mathbf{x ~ 1 0 ^ { 3 } )}\right.$ \\
\cline { 2 - 2 } Level 2 variances & \\
Plot & 9.80 \\
Species & 6.86 \\
Level 1 variance & 16.36 \\
Residual & \\
\hline
\end{tabular}

Table S3e: Mixed model predictions for leaf calcium per unit dry mass $\left(C a_{\mathrm{m}}: \mathrm{mg} \mathrm{g}^{-1}\right)$ as in Eqn 4, but with species affiliation (đ) treated as a fixed rather than a random effect.

\section{Fixed Effect}

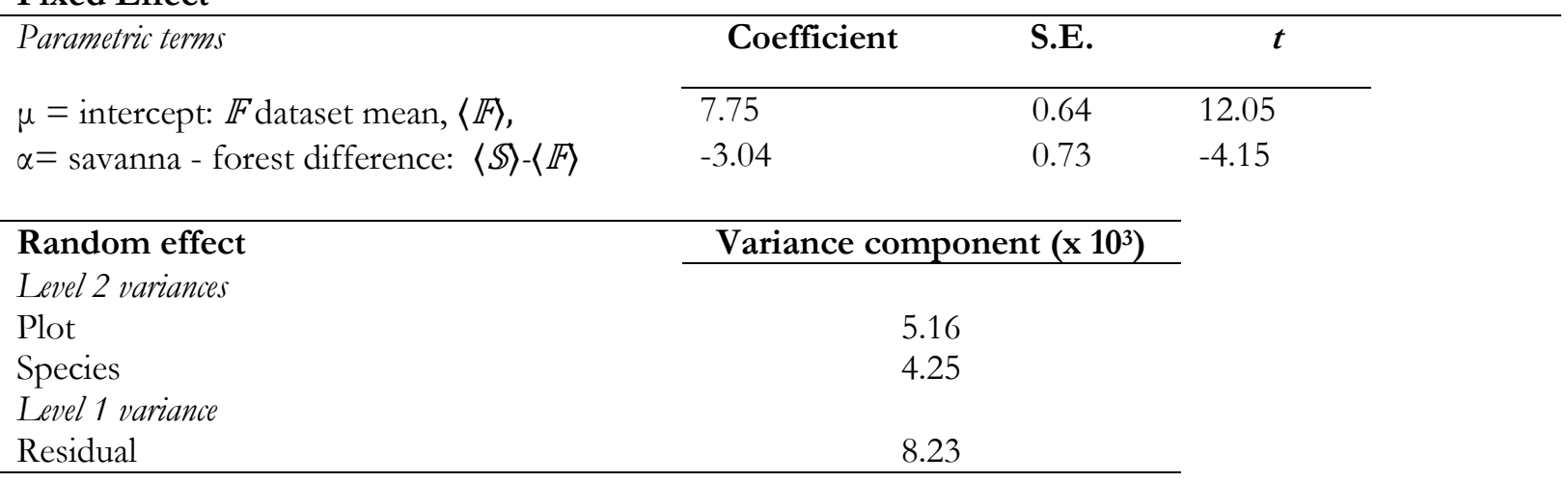

Table S3f: Mixed model predictions for leaf potassium per unit dry mass $\left(K_{\mathrm{m}}: \mathrm{mg} \mathrm{g}^{-1}\right)$ as in Eqn 4, but with species affiliation (ब) treated as a fixed rather than a random effect 


\section{Fixed Effect}

Parametric terms

\begin{tabular}{ccc} 
Coefficient & S.E. & $\boldsymbol{t}$ \\
\hline 2.91 & 0.27 & 10.80 \\
-0.61 & 0.32 & -1.94
\end{tabular}

$\mu=$ intercept: $\mathbb{F}$ dataset mean, $\langle\mathbb{F}\rangle$,

Variance component

\begin{tabular}{lr} 
Random effect & Variance comp \\
\cline { 2 - 2 } Level 2 variances & 0.87 \\
Plot & 0.83 \\
Species & 1.49 \\
Level 1 variance & Residual
\end{tabular}

Table S3g: Mixed model predictions for leaf magnesium per unit dry mass $\left(M g_{\mathrm{m}}: \mathrm{mg} \mathrm{g}^{-1}\right)$ as in Eqn 4 , but with species affiliation (đ) treated as a fixed rather than a random effect

\section{Fixed Effect}

\begin{tabular}{lccc}
\hline Parametric terms & Coefficient & S.E. & $\boldsymbol{t}$ \\
\cline { 2 - 3 }$\mu=$ intercept: $\mathbb{F}$ dataset mean, $\langle\mathbb{F}\rangle$, & 1.42 & 0.025 & 56.38 \\
$\alpha=$ savanna - forest difference: $\langle\mathscr{S}\rangle-\langle\mathbb{F}\rangle$ & 0.09 & 0.035 & 2.53
\end{tabular}

\begin{tabular}{lc}
\hline Random effect & Variance component $\left(\mathbf{x ~ 1 0 ^ { 3 }}\right)$ \\
\cline { 2 - 2 } Level 2 variances & 3.6 \\
Plot & 22.1 \\
Species & \\
Level 1 variance & 21.4 \\
Residual & \\
\hline
\end{tabular}

www.try-db.org/Mixed model predictions for leaf construnction costs $\left(K: \mathrm{mg} \mathrm{g}^{-1}\right)$ as in Eqn 4, but with species affiliation (đ) treated as a fixed rather than a random effect. 


\section{References}

Veenendaal, E, Torello-Raventos, M, Feldpausch, T, Domingues, TF, Gerard, FF, Schrodt, F, Saiz, G, Quesada, CA, Djagbletey, G, Ford, A, Kemp, J, Marimon, BS, Marimon Junior, BH, Lenza, E, Ratter, JA, Maracahipes, L, Sasaki, D, Sonké, B, Zapfack, L, Villarroel, D, Schwarz, M, Ishida, FY, Gilpin, M, Nardoto, GB, Affum-Baffoe, K, Arroyo, L, Bloomfield, K, Gjeca, G, Compaore, H, Davies, K, Diallo, A, Fyllas, NM, Gignoux, J, Hien, F, Johnson, M, Mougin, E, Hiernaux, P, Killeen, TJ, Metcalfe, D, Miranda, HS, Steininger, M, Sykora, K, Bird, MI, Grace, J, Lewis, SL, Phillips, OL, Lloyd, J (2014) Structural, physiognomic and aboveground biomass variation in savanna-forest transition zones on three continents. How different are co-occurring savanna and forest formations? . Biogeosciences Discussions 11, 4591 - 4636. 